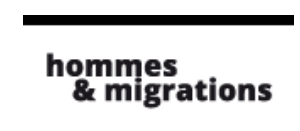

Hommes \& migrations

Revue française de référence sur les dynamiques

migratoires

1286-1287 | 2010

Les migrations subsahariennes

\title{
Les villages "multi-situés" sénégalais face à la nouvelle configuration migratoire mondiale
}

\section{Hamidou Dia}

\section{(apenEdition \\ Journals}

\section{Édition électronique}

URL : http://journals.openedition.org/hommesmigrations/1757

DOI : 10.4000/hommesmigrations. 1757

ISSN : 2262-3353

\section{Éditeur}

Musée national de l'histoire de l'immigration

\section{Édition imprimée}

Date de publication : 1 juillet 2010

Pagination : 234-244

ISSN : 1142-852X

\section{Référence électronique}

Hamidou Dia, "Les villages "multi-situés" sénégalais face à la nouvelle configuration migratoire mondiale », Hommes \& migrations [En ligne], 1286-1287 | 2010, mis en ligne le 29 mai 2013, consulté le 01 mai 2019. URL : http://journals.openedition.org/hommesmigrations/1757 ; DOI : 10.4000/ hommesmigrations. 1757 


\section{Les villages "multi-situés" sénégalais face à la nouvelle configuration migratoire mondiale}

Par Hamidou Dia, sociologue, Institut de recherche pour le dévelopement (IRD)

Les migrants originaires de la vallée du fleuve Sénégal développent des structures d'organisation en réseau dont le cœur est constitué par leur village de départ. Des associations sont créées en fonction des liens de parenté et de leur situation géographique. D'un point de vue social et économique, le partage d'une communauté

d'origine s'avère décisif à la fois dans la vie en migration et dans les sites de départ. Mais, confrontés à l'individualisation des parcours et à leur perte d'influence face à d'autres pouvoirs, ces espaces de solidarité sans frontière sont aujourd'hui menacés. 
Les migrations sont constitutives de l'identité du Sénégal contemporain. Multiformes et pluridimensionnelles, elles prennent leur source dans les zones rurales du pays. Aujourd'hui, elles concernent la totalité des régions de cet État sahélien ; néanmoins, les mobilités portent la marque de groupes précis et enracinés dans des territoires déterminés à partir desquels ils se déploient à travers le monde ${ }^{(1)}$. Cette dispersion se fait non seulement dans le cadre régional et national, mais aussi ouest-africain, continental, puis mondial. Il en est ainsi des Haalpulaaren, habitants de la moyenne vallée du fleuve Sénégal : contrairement à leurs célèbres voisins soninké, ressortissants pour la plupart de la partie amont, ceux-là sont restés relativement à l'abri de mouvements substantiels, au moins jusqu'à la fin des années trente, en raison principalement d'une sécurité alimentaire garantie par des terres fertiles à travers les cultures de décrue ${ }^{(2)}$. À la crise écologique, les populations apportent une réponse migratoire qui se décline d'abord par la recherche du numéraire dans les centres urbains sénégalais. Àpartir des années cinquante, d'autres destinations africaines sont explorées $^{(3)}$. Les années soixante et soixante-dix consacrent de nouveaux horizons comme la France. Les années quatre-vingt et quatre-vingt-dix donnent une dimension mondiale à ces mobilités qui trouvent un ancrage dans d'autres pays européens, en Amérique du Nord et en Asie ${ }^{(4)}$.

Ces migrations à partir des villages de la moyenne vallée du fleuve Sénégal ont fini, au bout d'un demi-siècle, par produire des univers de sens sur des territoires différents mais reliés par une commune référence à un site rural originel, que nous appelons des villages "multi-situés". Elles-mêmes engendrées par une pluralité de contextes socio-historiques, ces mobilités connaissent un nouvel âge fait de remise en questions sans précédent. De fait, la base de l'économie politique construite à partir de ces villages sénégalais est attaquée : les villages multi-situées appartiennent-ils alors à une ère révolue $e^{(5)}$ ?

\section{Le village multi-situé : scène sociale et univers de sens}

Le village multi-situé indique l'articulation entre le local, le régional, le national et l'international, il combine l'étude des lieux, des groupes d'appartenance et des réseaux. Ce concept comprend les habitants du site originel d'où sont partis des migrants, ainsi que les ressortissants vivant à Dakar, dans les grandes villes africaines d'installation ou de passage et dans les cités globales occidentales. Il implique les descendants des migrants, où qu'ils se trouvent dans le pays et dans le monde. Si la migration est au principe de sa constitution, il n'est cependant pas besoin d'émigrer pour en faire partie. 
C'est un espace de circulation d'hommes, d'idées, de paroles, de représentations, de pratiques, de biens et d'argent. Cette circulation est médiatisée par plusieurs institutions qui constituent le village comme référent : l'association globale et ses ramifications nationale et locale; les fêtes religieuses; les tournois sportifs et certains événements familiaux. Divers liens sont tissés entre le village d'origine et les sites sur lesquels il s'actualise par la présence de ses ressortissants ; des relations multiformes (amicales, conjugales, familiales, politiques, religieuses...) sont aussi nouées entre les différents territoires d'installation ou de passage des migrants. Le village "multi-situé" n'est pas une réalité statique. Le site originel est un lieu de vie, un espace de transformations et de réappropriations. Il ne peut être sérieusement assimilé à ce qu'il était avant le départ des pionniers de la migration; des institutions, des représentations et des pratiques perdurent, mais d'autres émergent. Les autres sites ne sont pas non plus une

\section{Le village multi-situé indique l'articulation entre le local, le régional, le national et l'international, il combine l'étude des lieux, des groupes d'appartenance et des réseaux.}

répétition communautaire à l'identique du village d'origine; ici, on n'a plus affaire à des "villages-bis" lovés dans des foyers de travailleurs. De la même manière qu'ils s'enracinent sur un territoire, les ressortissants en adoptent certaines pratiques; en s'installant en France, les migrants et leurs descendants s'engagent dans des processus qui ne trouvent leur intelligibilité que dans

le contexte français ; il en va de même pour ceux qui vivent à Dakar (Sénégal) ou à Libreville (Gabon), pour ne citer que ces exemples. De ce fait, en même temps qu'un référent commun autour du village est partagé, des logiques différenciées en fonction de la pluralité des lieux et des contextes nationaux voient le jour.

L'hétérogénéité est une caractéristique majeure du village multi-situé. Ce dernier est ainsi un complexe plurilinguistique: des praticiens du pulaar (langue de la région d'origine) y côtoient des locuteurs du wolof (principale langue de communication du Sénégal), du français (les enfants nés en migration), de l'américain, de l'italien ou encore du fang (langue du Gabon). Sous ce rapport, la période des grandes vacances, entraînant le retour au village de certains migrants, transforme le site originel en une gigantesque scène polyglotte: dans les concessions, les enfants parlent à la fois français, pulaar, wolof, anglais...; leurs prénoms sont rattachés à leur lieu et pays de provenance pour les différencier de leurs homonymes locaux: ainsi dira-t-on "Mariéta-Gabon, Amadou-France, Moussa-Washington, Aysata-Milan, Coumba-Dakar"... pour interpeller les uns et les autres. 


\section{Forces et faiblesses d'une structure réticulaire}

Le village multi-situé est aussi un conglomérat de nationalités. On y trouve des individus détenteurs d'une ou de plusieurs des nationalités suivantes : sénégalaise, mauritanienne, gabonaise, française, italienne ou américaine ; il en découle des conséquences importantes sur la capacité à circuler d'un site à l'autre, sur les possibilités éducatives, professionnelles, économiques des uns et des autres. En outre, le village multi-situé est une "alchimie de cultures" : cultures paysanne, nomade et islamique de la vallée du fleuve Sénégal ; cultures urbaines dakaroises et/ou occidentales; cultures des couches moyennes ou supérieures sénégalaises et/ou occidentales; cultures ouvrière, politique, syndicale françaises; cultures associatives, professionnelles, éducatives françaises et sénégalaises. Des influences diverses se rencontrent, se rejettent ou se fécondent en fonction d'événements et de situations sociales précises.

Le village multi-situé rassemble des individus de générations et d'âges différents, mariés comme célibataires. Il comprend également une pluralité de profils socioprofessionnels. On y trouve aussi bien des personnes n'ayant jamais été scolarisées que des ingénieurs ou des universitaires, des hommes politiques, des religieux, des artistes, des entrepreneurs, comme des chômeurs, des ouvriers ou des employés... Il est surtout l'image d'une interconnexion forte entre le rural et l'urbain, entre le national et l'international. Cette interconnexion entre divers ordres de réalités différenciés en fait un haut lieu de compétition distinctive : en tant que scène de sens pour tous les acteurs, une lutte pour le pouvoir multiforme y est à l'cuvre, ce qui n'autorise pas à en faire une donnée compacte, un bloc monolithique.

Entendu ainsi, le concept permet de rendre compte des migrations non seulement à partir de la moyenne vallée du fleuve Sénégal, mais aussi d'autres régions de ce vaste espace qu'est le Sahel. De ce fait, les migrations qui ont pour origine le nord du Sénégal ont produit de nombreux villages multi-situés : pour ce qui est du groupe spécifique des Haalpulaaren, ce concept s'avère heuristique pour analyser les mobilités à partir de l'actuelle région de Matam et du département de Podor. Aujourd'hui la viabilité de ces villages multi-situés en tant que collectifs de ressources et de protection, en tant que scènes sociales de reconnaissance et lieux de légitimation de pratiques et d'institutions les plus variées est fortement questionnée par deux séries de logiques : la première est inscrite dans le fonctionnement des associations qui le structurent en partie ; la seconde est à rapporter à des phénomènes plus globaux, aux effets complexes. 


\section{Les associations et l'orientation développementaliste : la fin d'une illusion?}

Les villages multi-situés sont constitués comme collectifs par leurs organisations, en particulier les associations qui rassemblent leurs ressortissants ${ }^{(6)}$. Sur tous les sites d'installation des ressortissants, des regroupements sont institués : dans un premier temps, ils prennent en charge des questions spécifiques aux territoires de résidence (hébergement, nourriture, logement, emploi, santé, frais liés aux décès). Ensuite, sous l'égide d'organismes de solidarité internationale et par l'opportunité juridique offerte par la loi 1901, qui permet aux migrants établis en France de se regrouper, une vision globale et formelle de ces regroupements épars s'affirme. Elle se traduit par la mue de ces entités en de grandes associations dotées de sections plus ou moins autonomes sur chaque site, y compris le village d'origine. Les actions d'entraide se transforment en adoptant souvent la problématique du développement. Les organisations prennent ainsi en charge des questions qui relèvent classiquement de la souveraineté de l'État, telles que l'éducation, la santé, l'hydraulique, voire la culture. Cette orientation est à l'origine de transformations dans les villages d'origine.

Néanmoins, la capacité des associations à agir en tant que collectifs et ressources s'amoindrit. Sur tous les sites urbains (Abidjan, Libreville, Pointe-Noire, Brazzaville, Dakar, Nouakchott, région francilienne, etc.) est à l'ceuvre un phénomène d'installation qui se traduit tout à la fois par un éparpillement dans la ville et un approfondissement générationnel : les regroupements dans des foyers ne sont plus la norme classique de logement; le célibat ou le couple à distance ne constituent plus les formes de conjugalité dominantes. De plus, les profils des membres ou des adhérents potentiels se diversifient: pour beaucoup, ils ont des trajectoires, des histoires et des intérêts forts différents.

Ces facteurs réduisent la vie des organisations. Les réunions deviennent de plus en plus difficiles à tenir; les orientations font l'objet d'âpres discussions; le recouvrement des cotisations est faible au regard du nombre de membres inscrits sur les listes. Par ailleurs, les partenariats institutionnels obéissent à des procédures qui requièrent des compétences techniques et administratives disponibles et qui sont souvent chronophages. Enfin, la communication entre les différents sites est parasitée par des luttes de préséance et l'omniprésence des nouvelles technologies de l'information et de la communication qui accentuent les capacités à manceuvrer à distance et à donner du poids aux positionnements factionnels ${ }^{(7)}$. À cela s'ajoute le fait que ces organisations inspirent de la méfiance aux hommes politiques locaux, qui les considèrent comme des viviers de personnel politique concurrent, en dépit des déclarations volontaristes de l'État sénégalais visant à capter les bénéfices que peuvent générer les migrations. 


\section{Le déficit d'engagement accroît la perte d’influence}

L'autonomisation des sites est l'autre grande logique qui travaille à l'affaissement du travail associatif. En effet, sur certains sites, la présence villageoise est plus que cinquantenaire (Dakar, région parisienne, Gabon). Sur d'autres, elle totalise au moins vingt ans d'installation, voire trente ou quarante ans (États-Unis, Italie, Égypte, Maroc). Chacun des sites constitue un espace propre, avec ses réalités. Sur chacun, les trajectoires divergent: différenciations professionnelle, résidentielle, statutaire qui continuent à affaiblir l'intérêt commun pour les villages d'origine. Ceux qui sont dotés de suffisamment de ressources (capitaux économiques, capitaux culturels, politiques, etc.) s'inscrivent dans une dynamique de mobilité sociale au sein des lieux d'installation. Si, sur tous les sites, les sections se renforcent au moins d'un point de vue formel par l'arrivée de nouveaux membres, l'engagement est rarement transmis auprès des descendants, tandis que toute une rhétorique est conçue et diffusée en ce sens. La participation, dans les cas où elle a 
lieu, réside dans l'acquittement occasionnel de cotisations mensuelles, bimensuelles ou trimestrielles, mais l'engagement au double plan de la conception des idées, des projets et de leur mise en pratique est faible.

Dans les villages d'origine, d'autres sources de pouvoir, de légitimité et de ressources voient le jour et se renforcent continuellement depuis une trentaine d'années, du fait, notamment, de la décentralisation promue par l'État du Sénégal ${ }^{(8)}$. Certes, les sections locales des associations internationales continuent à être fonctionnelles et développent des partenariats divers (associations de migrants, organisations non gouvernementales de développement, mutuelles d'épargne et de crédit), mais les figures qui les animent s'investissent davantage au niveau départemental, voire régional en tant que conseiller rural, conseiller régional, fonctionnaire contractuel local, ou encore par l'accès aux crédits octroyés par des organismes publics étatiques ou parapublics de financement ${ }^{(9)}$.

Linvestissement dans la vie politique nationale ou dans des organisations non gouvernementales externes permet de s'affranchir de la tutelle de certains segments de la parenté représentés par les migrants qui agissent, via l'organisation villageoise multi-située, comme bailleurs de fonds. En effet, pour sauver la face, il est toujours préférable d'être redevable envers des institutions ou des individus extérieurs au village multi-situé plutôt qu'envers des migrants avec lesquels on est en concurrence symbolique car ils appartiennent au même groupe de parenté ou au même groupe d'âge. Vis-à-vis des membres du village multi-situé, une réussite basée sur des contacts noués avec des étrangers est davantage perçue comme un succès personnel.

\section{Reflux des mobilités internationales et densification de scènes sociales spécifiques}

Mais d'autres facteurs externes compliquent le tableau : les associations peuvent faiblir, alors même que le village multi-situé se renforce en tant que scène sociale du quotidien. Si les villages multi-situés se sont constitués sur la base des migrations depuis cinquante ans, l'inflation législative au Nord sur les conditions d'entrée et de séjour empêche le renouvellement des sites européens. Or, ce sont parmi les plus importants au plan économique, en ce qu'ils constituent des lieux d'accumulation d'où les ressortissants peuvent contribuer aux transformations de la vie du village, en l'absence d'État-providence au Sénégal.

Certes, l'essentiel des mouvements migratoires se déroule en Afrique ${ }^{(10)}$, et les ressortissants de ces zones ont fortement contribué aux transformations de la 
vallée; néanmoins, ce sont les migrants résidant en Europe qui restent les plus stables. Cependant, depuis une trentaine d'années, avec un pic atteint dans la première décennie du nouveau millénaire, de nouvelles orientations sont prises par les pouvoirs publics, notamment en France: promotion de la "migration choisie"; durcissement des conditions du regroupement familial, considéré comme une source illégitime de migration ; mise en place et renforcement de dispositifs d'incitation au retour dans les pays d'origine, à travers la politique dite "du codéveloppement". Enfin, des accords dits "de gestion concertée" sont signés avec certains pays, en particulier le Sénégal, afin d'impliquer les États d'origine des migrants dans le travail de contrôle des mobilités de leurs ressortissants ${ }^{(11)}$.

Ces dispositifs législatifs et institutionnels tendent vers une logique d'endiguement et sont sous-tendus par une rhétorique de stigmatisation des populations en provenance de l'Afrique subsaharienne. De fait, les renouvellements sur les sites européens des villages multi-situés se font de plus en plus

\section{Chacun des sites constitue un espace propre, avec ses réalités. Sur chacun, les trajectoires divergent : différenciations professionnelle, résidentielle, statutaire qui continuent à affaiblir l'intérêt commun pour les villages d'origine.} rares et deviennent extrêmement coûteux pour les candidats à l'émigration. Ainsi, la circulation entre les sites est de plus en plus unilatérale : seuls les détenteurs d'un passeport européen ont un accès physique sur les autres territoires d'installation, une entorse grave au droit à la mobilité(12). La raréfaction des arrivées sur les sites du Nord produit des effets sur la capacité des associations de ressortissants, mais aussi sur l'économie domestique transnationale ${ }^{(13)}$.

Un des effets collatéraux de cette évolution législative et réglementaire est d'accentuer, sur des lieux particuliers, la "sur-sollicitation" de la communauté pour des aspects les plus divers : logement, emploi, mariage, etc., en l'absence de garanties universelles et neutres de l'État, qui ne cesse de réaffirmer son caractère républicain de façon désincarnée ${ }^{(14)}$. Alors que les profils arrivant dans les années deux mille sont bien plus qualifiés et citadins que les pionniers de la migration internationale, les difficultés administratives et matérielles les incitent à un retour contraint dans la communauté qui assure leur protection.

Ainsi, l'évolution des associations villageoises témoigne-t-elle des "âges de la migration" mis en évidence par Sayad ${ }^{(15)}$ : au fil des générations, la divergence des trajectoires éloigne d'un investissement fort envers le pays d'origine. Mais, à l'inverse, les villages multi-situés prennent une importance croissante en tant que scène sociale du quotidien. Alors qu'il est difficile à ces nouveaux migrants de faire valoir 
leurs compétences scolaires et culturelles sur les marchés du travail du Nord, ils trouvent un intérêt matériel et symbolique à user de leur "capital d'autochtonie ${ }^{(16)}$ " au sein du village transnational.

\section{De nouvelles limites à la circulation en Afrique}

Sur le versant africain des villages multi-situés, les circulations sont soumises à de fortes contraintes politiques et économiques. En effet, pendant près de quarante années, l'Afrique centrale s'est révélée comme l'une des destinations phares des migrants du fleuve. Néanmoins, les guerres civiles dans les deux Congo et leurs corollaires ont amoindri les mouvements vers ces États ${ }^{(17)}$, alors même que le Gabon, sous l'effet du relatif tarissement de la manne pétrolière et de la crise de l'emploi local, élève les exigences en matière de séjour des étrangers, à travers une hausse continue du prix des titres de séjour. L'Afrique du Sud, objet de convoitises pendant les premières années post-apartheid, met un bémol aux ambitions des candidats au séjour sous l'effet de poussées xénophobes ${ }^{(18)}$. En Afrique de l'Ouest, le premier pays d'accueil des étrangers, la Côte d'Ivoire, connaît des turbulences identitaires depuis la popularisation du concept d'“ivoirité", mettant ainsi les étrangers en situation inconfortable au double plan du séjour et de l'emploi ${ }^{(19)}$. En Afrique du Nord, les possibilités s'amoindrissent aussi en dépit de connexions multiformes entre le sud et le nord du Sahara : d'abord, le Maghreb est devenu un poste de contrôle avancé des frontières européennes, notamment de l'accès aux côtes espagnoles et à l'Europe du sud de manière générale ${ }^{(20)}$; ensuite, le commun partage de la religion musulmane ne suffit pas à faciliter l'acceptation par les populations autochtones de migrants subsahariens stigmatisés et rendus responsables de la diffusion locale de certains maux $^{(21)}$. Si les relations avec la Mauritanie voisine commencent à se normaliser, les populations riveraines du fleuve Sénégal n'en sont pas encore au stade d'exploiter leurs terres situées sur la rive droite en raison de la persistance de la mémoire des événements survenus à la frontière entre les deux pays à la fin des années quatre-vingt ${ }^{(22)}$.

De fait, ce sont tous les lieux de destinations, classiques et nouveaux, qui semblent ou momentanément ou durablement fermés au renouvellement de la migration et à la viabilité d'une économie politique construite par les mobilités. Pendant ce temps, sur les sites originels des villages multi-situés, un système de reproduction basé sur d'autres sources de revenus peine à se mettre en place. Mieux, retournent dans ces endroits d'anciens migrants en Afrique centrale victimes d'expulsion ou en situation 
d'échec, des jeunes sans perspectives d'insertion professionnelle en ville ou encore d'anciens pensionnaires des écoles coraniques sans qualifications mobilisables sur le marché du travail national, dans un contexte de désaffection profonde des populations à l'égard de l'agriculture irriguée promue par l'État sénégalais au début des années soixante-dix ${ }^{(23)}$. Il faut cependant noter que se dessine timidement l'urbanisation de quelques bourgs de la vallée : les obstacles aux migrations internationales ont tendance à intensifier les mouvements régionaux. Ainsi, le village multi-situé a une importance forte pour les migrants, mais les circulations au sein de cet espace transnational sont de plus en plus réservées aux individus et aux familles qui ont pu, sur plusieurs générations, accumuler des ressources diverses.

\section{Conclusion}

Face au reflux de l'économie agricole des années trente, à la diffusion du numéraire dans les années cinquante, aux crises écologiques des années soixante et soixantedix, aux effets calamiteux de la faillite des politiques publiques post-coloniales de modernisation industrielle et agricole des années quatre-vingt, aux conséquences multiformes des politiques d'ajustement structurelles, à la diffusion du chômage des qualifiés et des diplômés de l'enseignement supérieur et à la saturation des ménages urbains, les Sénégalais, surtout ceux qui sont originaires des zones rurales, notamment au nord du pays, ont élaboré des solutions de réponse toutes structurées par la migration.

Le phénomène finit par constituer un des marqueurs majeurs de l'identité de l'actuel Sénégal. Au moment même où l'État local commence à prendre au sérieux ses ressortissants établis à l'extérieur sous l'effet des politiques interventionnistes du Nord, à travers entre autres, la politique de codéveloppement qui sublime de façon factice le rôle des migrants présentés sous le "label" de diasporas, après la découverte subite de la manne financière, intellectuelle ou technique qu'ils représentent, les fondements même de la participation des migrants aux transformations du Sénégal sont compromis par les coups de butoir des dispositifs législatifs et institutionnels. De plus en plus, le village multi-situé constitue surtout pour les migrants une scène sociale à l'importance symbolique et matérielle, davantage qu'un acteur de transformation des localités d'origine. Les villages du nord du Sénégal, qui se sont dupliqués sur des territoires très différents, sont ainsi à la croisée des chemins : entre une externalisation devenue problématique et un État local incapable de les protéger, fût-il décentralisé. En conséquence, le local globalisé interpelle l'instance nationale dans sa capacité à intégrer toutes ses composantes. 


\section{Notes}

1. Voir Diop Amadou Moustapha, Tradition et adaptation dans un réseau de migration sénégalais. La communauté manjak en

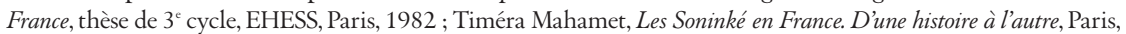
Karthala, 1996 ; Mboup Mourtala, Les Sénégalais d'Italie, Paris, L'Harmattan, 2000 ; Kane Abdoulaye "Senegal's Village Diaspora and the People Left Ahead", in Bryceson Deborah, Vuorella Ulla, The Transnational Family, New York, Berg, 2002, pp. 245-263.

2. Lericollais André, Vernière Marc, "L'émigration toucouleur : du fleuve Sénégal à Dakar", in Cahiers de l'ORSTOM, série sciences humaines, ${ }^{\circ}$ 2, vol. XII, 1975, pp. 161-175.

3. Bredeloup,Sylvie, La Diams'pora de la vallée du fleuve Sénégal, Toulouse, Presses universitaires du Mirail, 2007.

4. Dia Hamidou, "Villages multi-situés du Fouta-Toro en France : le défi de la transition entre générations de caissiers, lettrés et citadins", in Asylon(s), n 3, 2008, www.terra.rezo.net/article713.html.

5. Cette contribution se fonde des recherches de terrains conduites entre 2003 et 2010 qui se déploient à partir de la moyenne vallée du fleuve Sénégal. Ces enquêtes ont été menées en Europe, en Amérique du Nord et en Afrique dans le cadre d'une thèse de doctorat et d'un projet de recherches sur les impacts territoriaux des migrations africaines. Voir Dia Hamidou, Espaces domestiques, espaces villageois, espaces urbains multi-situés : cinquante ans de migrations à partir de la moyenne vallée du fleuve Sénégal, thèse de doctorat en sociologie, Paris, université Paris-Descartes, 2009 ;

Dia Hamidou, "Les transferts d'argent des migrants de la vallée du fleuve Sénégal. Processus d'affectation et impacts sur les villages d'origine", in Document de synthèse des projets du programme FSP $n^{\circ}$ 2003-74 Migrations internationales, recompositions territoriales et développement en Afrique, IRD éditions, 2009, pp. 25-36.

6. Voir Lavigne-Delville Phillipe, Irrigation, migration et stratégies paysannes dans la vallée du fleuve Sénégal, Paris, Syros Alternatives, 1991 ; Quiminal Catherine, Gens d'ici, gens d'ailleurs, Paris, Christian Bourgois, 1991 ; Dia, Hamidou, "Les ressources d'une diaspora de la moyenne vallée du fleuve Sénégal", in Diop, Momar Coumba (Dir.) Le Sénégal des migrations : mobilités, identités et sociétés, Karthala, Paris, 2008, pp. 179-194.

7. Dia Hamidou, "Le téléphone portable dans la vallée du fleuve Sénégal", in Agora, n 46, 2007, pp. 70-80.

8. Copans, Jean, Développement mondial et mutations des sociétés contemporaines, Paris, Armand Colin, 2006 ;

Diop Djibril, Décentralisation et gouvernance locale au Sénégal. Quelle pertinence pour le développement local ?, Paris, L'Harmattan, 2006.

9. Dahou Tarik, Entre parenté et politique. Développement et clientélisme dans le Delta du Sénégal, Paris, Karthala, 2005.

10. Daum Christophe, Dougnon Isaïe, "Les migrations internes au continent africian", in Hommes et Migrations, $n^{\circ} 1279,2009$, pp. 6-11.

11. Rodier Claire, Terray Emmanuel, Immigration : fantasmes et réalités. Pour une alternative à la fermeture des frontières, Paris, La Découverte, 2008 ; Spire Alexis, Acueillir ou reconduire. Enquête sur les guichets de limmigration, Paris, Raisons d'agir, 2008.

12. Withol de Wenden Catherine, "Pour un droit à la mobilité et une démocratisation des frontières", in Panoramiques, $\mathrm{n}^{\circ} 55,2001$, pp. 8-14.

13. Dia Hamidou, "Le téléphone portable dans la vallée du fleuve Sénégal", op. cit.

14. Ndiaye Pap, La Condition noire, Paris, Calmann-Lévy, 2008.

15. Sayad Abdelmalek, "Les trois âges ou l'émigration algérienne en France", in Actes de la recherche en sciences sociales, $\mathrm{n}^{\circ} 15,1977$, pp. 59-81.

16. Retière Jean-Noël, "Autour de l'autochtonie : réflexion sur la notion de capital social populaire", in Politix, $\mathrm{n}^{\circ}$ 63, 2003, pp. 121-143.

17. Yengo Patrice, La Guerre civile au Congo-Brazzaville, 1993-2002. "Chacun sa part”, Paris, Karthala, 2006.

18. Whitehouse Bruce, "Migrants et insécurité existentielle. Le cas de Brazzaville", in Hommes et Migrations, $n^{\circ} 1279$,

2009, pp. 80-87.

19. Bredeloup Sylvie, "Les Sénégalais de Côte d'Ivoire face aux redéfinitions de l'ivoirité", in Études et Migration, XXXIII, n 121, 1996.

20. Pian Anaïk, Aux nouvelles frontières de l'Europe. L'aventure incertaine des Sénégalais au Maroc, Paris, La Dispute, 2009.

21. Timéra, Mahamet, Faire et défaire les identités. Migration, ethnicité, "race" et religion, 2 volumes, Paris, HDR, 2008.

22. Voir Leservoisier, Olivier, La Question foncière en Mauritanie. Terres et pouvoirs dans la région du Gorgol,

Paris,L'Harmattan, 1995 ; Vandermotten Céline, Géopolitique de la vallée du fleuve Sénégal, L'Harmattan, Paris, 2005 ; Frésia Marion, "De l'exil au retour : les enjeux du rapatriement des Mauritaniens réfugiés au Sénégal", in Asylon(s), $\mathrm{n}^{\circ}$ 3, 2008, www.terra.rezo.net/article710.html.

23. Crousse Bernard, Mathieu Paul, Seck Sidi Mohamed (dir.), La Vallée du fleuve Sénégal. Une décennie d'aménagements, Paris, Karthala, 1991. 
